\title{
Review on strategies to minimize the appearance of multi-drug resistant organism
}

Professor Alberto Frutos Pérez-

Surio ${ }^{1,2}$

Juan Gastón Añaños ${ }^{3}$,

Elisa Sahún García ${ }^{3}$,

Gema Sancho Monllor ${ }^{4}$

${ }^{1}$ Pharmacy Department. University Clinical Hospital Lozano Blesa

Zaragoza, Spain

${ }^{2}$ Microbiology, Preventive

Medicine and Public Health

Department

University of Zaragoza.

${ }^{3}$ Pharmacy Department

Barbastro Hospital

Barbastro, Spain

${ }^{4}$ Documentation and Information

Systems

Atlantis Project

Zaragoza, Spain

DOI:10.29251/ijpph.201874

${ }^{*}$ Corresponding author:

Professor Alberto Frutos Pérez-

Surio

Pharmacy Department

University Clinical Hospital

Lozano Blesa

Avenida San Juan Bosco 15, 50009

Zaragoza España

E-mail: ajfrutos@salud.aragon.es

\begin{abstract}
Background: The use of antibiotic drugs (ABX) in hospitals, and especially in intensive care units (ICU), is widespread. The early administration of $A B X$ therapy can improve survival rates. The influence and impact of the ABX are observed in the patients who receive them (clinical response, course) and in the ecosystem surrounding the patient (hospital flora).

Aim of the review: The objective of this review is to identify strategies that reduce or limit the appearance and transmission of multidrug-resistant microorganisms. This identification can then develop a rational use of the $A B X$ plan in the ICU.
\end{abstract}

Method: The following databases were queried; Medline, Embase, The Cochrane Library, and the Centre for Reviews and Dissemination (University of York), asking the questions in PICO format to evaluate the efficacy and safety of several interventions: A) Therapeutic de-escalation; B) Cycling of $A B X$ and; C) Early antibiotic treatment.

Results: A) In therapeutic de-escalation of 98 studies identified, three studies that met the inclusion criteria were analyzed. B) Two studies comparing antibiotic cycling versus other interventions were selected. C) No studies have been found with sufficiently robust methodological designs that address the ABX early treatment. There is no strong evidence to indicate which of the different antibiotic interventions (therapeutic de-escalation, cycling of $\mathrm{ABX}$ and preemptive treatment) is more effective in reducing antibiotic resistance in ICU patients. There is insufficient evidence that de-escalation of antimicrobial agents is effective against resistance. In patients admitted to the ICU with a low prevalence of 
fluoroquinolones resistance, increased exposure to this class of antibiotics, using antibiotic cycling, increases the emergence of resistant strains.

Conclusion: Despite the fact that no prospective studies were identified in this SR, rationale and day-to-day clinical practice experience suggest that multidisciplinary participation of different specialists in ABX's Infection and Policy Commission (or the ABX Commission), or the Pharmacy and Therapeutics Committee, might improve the development and application of these strategies.

Keywords: Drug resistance, Cross infection, Anti-bacterial agents, Intensive care units

\section{Introduction}

The use of antibiotic drugs (ABX) in hospitals, and especially in intensive care units (ICU), is widespread. The early administration of ABX therapy can improve survival rates (Mok et al., 2014). The influence and impact of the ABX are observed in the patients who receive them (clinical response, course) and in the ecosystem surrounding the patient (hospital flora). This impact is especially visible in the critically ill patients in the endemic flora of the ICU (Alvarez Lerma et al., 2010).

Treatment with broad-spectrum $\mathrm{ABX}$ is generally used for early treatment, since it has been shown that preemptive treatment, with the appropriate $A B X$, reduced mortality rates. This approach may expose individuals to excessive use of $\mathrm{ABX}$ and therefore, the selection of resistance to these pathogens (Silva et al., 2013).The European Centre for Disease Prevention and Control (ECDC) and the European Medicines Agency (EMA) estimated that each year 25,00o Europeans die as a direct consequence of a multidrugresistant infection (ECDC/EMA, 2009).
In Spain, as some projects in other European Member States (e.g. the Netherlands (Schuts et al., 2016)), the National Action Plan on Antimicrobial Resistance (AEMPS, 2018) was nationally coordinated in 2014 by the Spanish Agency of Medicines and Medical Devices, together with six Ministries, with the objective of minimizing the impact of antimicrobial resistance and how to be addressed jointly from the human and veterinary health. The importance of the $\mathrm{ABX}$ as part of the pharmacotherapy arsenal is unquestionable, hence the importance of improving the management of the knowledge about them.

Health and social care practitioners have to make clinical decisions daily. Compatible update of knowledge and health care is a complicated task, and Clinical Practice Guidelines (CPG) are useful tools to facilitate decision-making. Initiatives such as the Program for Optimizing the use of Antimicrobials (PROA) in Spanish hospitals (Rodriguez-Bano et al., 2012) can contribute to improve policy of $\mathrm{ABX}$, and minimize the emergence of resistance in microorganisms. Within the framework of the ICU it emphasizes the "Zero resistance" programme (Garnacho Montero et al., 2015).

One of the lines of work, typically proposals from hospital pharmacy services, is the sequential therapy, but there are interventions whose evidence is not proven and that should be evaluated. These include the therapeutic de-escalation, and cycling of $\mathrm{ABX}$ and preemptive treatment. The objective of this review is to identify strategies that reduce or limit the appearance and cross transmission of multidrug-resistant organisms in order to make rational use of the $\mathrm{ABX}$.

\section{Data and Methods:}

Strategies to minimize the emergence of multidrug-resistant organisms in ICU with policy measures of $\mathrm{ABX}$ that address: $\mathrm{A}$ ) 
Therapeutic de-escalation, B) Cycling of ABX, and C) Early antibiotic treatment. Other strategies, to reduce cross transmission of multidrug-resistant organisms in ICU, are also addressed, such us: 1) preventive isolation or 2) active surveillance for patients in the early identification of Methicillin-resistant Staphylococcus aureus (MRSA) on asymptomatic carriers of other multidrug-resistant microorganisms.

Therefore, this review is focused to identify interventions that might help to prevent resistance to antimicrobials, in the hospital and ICU, to reduce morbidity and mortality by antimicrobial-resistant organisms.

The following databases were searched until January 2018: Medline (via PubMed), Embase, The Cochrane Library and the Centre for Reviews and Dissemination, and University of York. We used the controlled vocabulary thesaurus (MeSH or Emtree), used for indexing articles for PubMed, Cochrane and CRD (MeSH) or Embase (Emtree), as appropriate, as well as searching by free language terms for each construct of the research question, disaggregated in its PICO form (Patient, Intervention, Comparison, and Outcome). Comprehensive and updated (2008-2018) search strategies, including comprehensive search terms (i.e. resistance antibiotic AND intensive care unit). The literature search, (can be found in the supplementary material, which is available to authorized users) with the ATC classification group Jo1 (WHO, 2017), is described in the flow diagram in Figure 1. The results were also accompanied by a manual search on UpToDate (Waltman, MA) and grey literature findings.

Inclusion and exclusion criteria were established a priori. We have included studies, which related interventions allowing estimators or comparative information on reduction, elimination of the appearance, or cross transmission of multidrugresistant microorganisms to ABX. Studies reporting interventions on reducing resistance levels, for various classes of antibiotics, such as aminoglycosides or quinolones, were excluded.

Systematic reviews (SR) that do not provide quantitative data on the results of the interventions of interest and articles without abstract were excluded. It is used as filter design studies, selecting those which are clinical practice guidelines (CPG), randomized clinical trials (RCT), and economic evaluation studies.

\section{Results}

\section{A) De-escalation therapy}

Of 98 identified studies, we have analyzed 3 studies that met the criteria of inclusion: 1 SR (Silva et al., 2013), 1 RCT (Kim et al., 2012), and 1 CPG (Dellinger et al., 2013). The SR, despite identifying several studies, discards them for being uninteresting interventions or clinical conditions not relevant to the review.

The study done by Kim et al., 2012 shows statistically significant differences in favour of de-escalation in terms of the adequacy of the $A B X$ and against the de-escalation in the time of occurrence of multi-drug resistant organism, primarily due to MRSA; $\mathrm{HR}=3.84$ (95\% CI: 1.06; 13.9). Mortality, duration of the ABX, stay in ICU or adverse treatment, showed no statistically significant differences. Dellinger et al., 2013 were advised to follow the principles of the Grading of Recommendations Assessment, Development and Evaluation (GRADE) system to guide assessment of quality of evidence, and include in the international CPG: 1 ) the need to identify pathogens and apply more efficient ABX treatment, 2) reduction of the spectrum of antibiotic coverage, and 3) the reduction of the duration of the ABX treatment.

This decreases the chances that the patient develops infections by multi-resistant pathogens and the beginning of a pattern of broad-spectrum 
treatment $\mathrm{ABX}$ to increase the effectiveness of the treatment and improve health outcomes.

\section{B) Cycling of ABX}

Two studies were selected (Nijssen et al., 2010; Martinez et al., 2006) that compare $A B X$ cycling to other interventions. The first, a period of cycling's three months, gets 14 crops with Enterobacteriaceae resistant to third generation cephalosporin's (3GCRE) for every 1,0oo patients per day to risk. This data is the same as during the baseline period, observation of the ICU. During the three months of not cycling (reducing exposure to beta-lactams and introduction of fluoroquinolones), there are 18 crops with (CPG) for every 1,ooo patients per day to risk. During the three months of cycling, isolated $2.5 \quad(\mathrm{CPG})$ resistant to fluoroquinolones (FRE) for every 1,00o patients per day to risk, being the similar results of the baseline period (2.1 FRE 1,00o patients day to risk) in contrast with the period of not cycling, which they have 8.3 FRE for every 1,0oo patients per day at risk (Nijssen et al., 2010).

When the explanatory model is adjusted by type of ICU, age of the patient, gravity - APACHE II, indication of admission, and reason of contacts, not cycling for 3 months in ICU (reduction of beta-lactam and introduction of fluoroquinolones), increases the speed of appearance of FRE in 4.1 times with regard to the cycling of $\mathrm{ABX}$ (adjusted HR $=4.1$ [95\% CI: 1.4; 11.9]) (Nijssen et al., 2010). The Group of Martinez et al. (2006) analyzed along 8 months, in two ICU, a pattern of cycling of $A B X$ with another mixed pattern that was administered to patients, as they enter consecutively to analyze the emergence of resistance in gram-negative enteric Bacilli.

They found that the prevalence of resistance acquired during two periods of study was highest during the mixed period than during the period of cycling ( $9 \%$ vs. $3 \%$ respectively, $\mathrm{p}=0.01$ ) for Pseudomonas aeruginosa and specifically, for cefepime. No statistically significant differences for other bacilli can be seen on Enterobacteriaceae (Martinez et al., 2006).

\section{C) Preemptive treatment}

No studies have been found with robust enough methodological designs that address the preemptive treatment. Study designs were not enable to provide robust evidence about preemptive treatment (De Waele et al., 2003; Piarroux et al., 2004).

\section{Cross transmission}

We found 5 studies, of which 3 are SR (Lam et al., 2012; Pammi et al., 2011; Backman et al., 2008) and 2 RCT (Huang et al., 2013; Climo et al., 2013). A SR evaluates the effectiveness of the measures of oral hygiene in reducing colonization of Staphylococcus aureus (Lam et al., 2012) and given that only 3 of the 15 studies included in the review had been done with ICU patients with mechanical ventilation (MV), results of the three studies were displayed (Pedreira et al. 2009; Scannapieco et al., 2009; Fourrier et al., 2000) with broader objectives.

In all three studies, part of the intervention consists of performing oral hygiene with chlorhexidine in critically ill patients with MV. Results from the Paediatric ICU show no difference on colonization of oropharyngeal, duration of the MV, length of stay in ICU, or isolation of multidrug-resistant bacteria (Pedreira et al. 2009). A second study with adult patients showed a significant reduction in the number of isolates by culture Staphylococcus aureus versus placebo at 2 and 4 days of admission to ICU with MV. There was no statistically significant difference in the occurrence of pneumonia, or it has been observed that interventions at the time delayed the onset of pneumonia 
(Scannapieco et al., 2009).

The work done in France excludes study to toothless patients and select patients with exclusively medical processes all with MV. Oral isotonic serum bicarbonate and oropharyngeal suction cleaning 4 times a day improve in a statistically significant way to more nosocomial infection (NI) that the application of gel with chlorhexidine associated with $0.2 \%$ in teeth and gums with sterile glove 3 times daily $(p=0.018)$. The application of chlorhexidine is also associated with a reduction in the number of ventilation-associated pneumonia (VAP) (p $<0.05$ ) (Fourrier et al., 2000).

The SR of Pammi et al. 2011 sought to analyze the effect of isolation measures in reducing the cross transmission of Candida from infants colonized or infected. No evidence was found supporting or not of isolation measures, both individually and in group, in ICU of infants (Pammi et al., 2011).

A SR identified which aims to evaluate the relationship between hand hygiene interventions and the incidence of NI (Backman et al., 2008). In addition to 35 studies, one included is a RCT in Paediatric ICU selected (except kidney) solid organ transplant patients. Observed hand washing with chlorhexidine before and after each patient contact or the use of robe and latex gloves non sterile with each patient contact, reduce the incidence of NI ICU in a statistically significant way between the previous year and the 6 months after the interventions (4.9/100 days vs. 2.2/100); $\mathrm{p}=0.0004$. Statistically significant differences were not found in NI average in transplanted between groups (Slota et al., 2001).

Work done with 74 ICU adults in the United States, included 74,256 patients and compared between different interventions (screening and isolation, specific decolonization and universal decolonization) to reduce the transmission of MRSA and other pathogens acquired in ICU. To compare interventions with the baseline period (prior to the implementation of interventions), a statistically significant reduction is observed of positive cultures for MRSA acquired in ICU ( $\mathrm{p}=0.01)$, as well as bacteraemia caused by others pathogens acquired in ICU ( $p<;$ o.0o1). Statistically significant differences for bacteraemia by MRSA acquired in ICU are not observed both to compare between periods and between groups. Comparing between groups, universal decolonization was the intervention that reduced the occurrence of positive cultures for MRSA and ICUacquired bacteraemia by other pathogens more effectively (Huang et al., 2013).

A multicenter RCT, with the participation of 9 ICU (a total of 7,727 patients) assessed the utility of body hygiene through cleaning wipes, with chlorhexidine gluconate $2 \%$, to reduce the risk of acquiring multiresistant organisms and bacteraemia nosocomial in critically ill patients. The duration of the intervention period decreased, in a statistically significant way, a $23 \%$ NI MRSA and Vancomycin-resistant Enterococcus (VRE), a $28 \%$ nosocomial bacteraemia, $31 \%$ primary bacteraemia, and 53\% bacteraemia, associated to central venous catheter. A reduction of $90 \%$ of fungaemia is also described, associated with central venous catheter (Climo et al., 2013).

Antimicrobial stewardship guidelines and initiatives previously published by the Infectious Disease Society of America (IDSA), e.g. IDSA and Society for Healthcare Epidemiology of America (SHEA) jointly published Guidelines for Developing an Institutional Program to Enhance Antimicrobial Stewardship, in Clinical Infectious Diseases (January 2007 vol. 44 no. 2 159-177) for developing institutional programs to enhance antimicrobial stewardship, an activity that includes appropriate selection, dosing, 
route, and duration of antimicrobial therapy. A brief summary related to "Infections and antimicrobial resistance in the intensive care unit: Epidemiology and prevention", found on UpToDate, is adapted with permission on table 1: Summary of recommendations for preventing ventilator -associated pneumonia (VAP) in adult patients (SHEA/IDSA).

\section{Discussion}

Studies of quality capable of sustaining analyzed ABX policy measures have been searched and identified. It would be necessary to design prospective research studies that can establish a causal relationship between the policy measures of $\mathrm{ABX}$ and the emergence of multidrug-resistant organisms in ICUs reduction. Studies with more robust designs that allow you to determine if oral hygiene in critically ill patients with MV's more 48 hours has a clinically relevant impact is required. As antimicrobial stewardship Pharmacist, you can make prospective audit and feedback, if you review the information and recommend that the prescribing team narrow therapy.

Antibiotics should be discontinued within 48 hours after surgery. It is necessary to carry out studies that allow determining the clinical impact of the role of isolation measures in patients, colonized or infected by Candida. There is no doubt of the importance of interventions to improve hand hygiene to reduce the incidence of NI in critically ill patients. Universal decolonization in critically ill patients reduce bacteraemia, prevents the realization of surveillance test, reduces the number of isolates of contact, and decreases the chances of transmission of infection to other patients. The personal hygiene of critically ill patients with chlorhexidine-impregnated wipes reduces the transmission of bacteria resistant to other patients.
Therapeutic de-escalation consists in initiating ABX therapy with broad-spectrum empirical therapy, with the aim of increasing the chances of covering the probable infectious agent. Subsequently, a broad spectrum treatment change is performed. De-escalation should occur with respect to culture data or clinical judgment. Empiric use of combination therapy should not be administered for longer than 3-5 days if de-escalation to a single agent is appropriate. Empiric antimicrobials should cover likely pathogens according to suspected location of infection and risk of multidrug-resistant pathogens. Other considerations in choosing appropriate antimicrobials include the patient's history of drug allergy or intolerance, recent antibiotic use, comorbidities, and antimicrobial susceptibility patterns in the community and hospital. Consider discontinuing antimicrobials in 7-10 days unless there is slow response, undrainable foci, immunosuppression, or multidrugresistant pathogens.

Blood cultures will be negative in most patients, despite a bacterial of fungal origin of sepsis. Clinical judgment is needed when considering discontinuation of antimicrobials. According to the results of the microbiological studies, a treatment with a more adjusted antimicrobial spectrum by one of the following two pathways: 1) change of $\mathrm{ABX}$, and; 2) Interrupt an antibiotic combination. An additional strategy to shorten the duration of ABX treatment (Alvarez Lerma et al., 2010; Silva et al., 2013). Crop performance is a prerequisite for the use of antibiotic de-escalation in critically ill patients, although the decision to reduce the intensity of treatment should be based on the patient's clinical course (Silva et al., 2013). Therapeutic de-escalation aims to achieve adequate antibiotic coverage through the use of $A B X$ in a targeted manner, reducing the spectrum of empirical coverage and reducing selective 
pressure on the patient's flora as well as the ecosystem in which the latter remains.

The cycling of ABX consists of the determined alternation of $\mathrm{ABX}$ by which the use of an $A B X$ or specific $A B X$ class is restricted for a certain period of time to be reintroduced subsequently (Sandiumenge et al., 2003). Other authors describe cycling as the periodic substitution of one class of $A B X$ by another class or the combination of ABX that present a spectrum of similar activity, but do not share the same mechanism of resistance (Alvarez Lerma et al., 2010). It is intended to minimize the occurrence of bacterial resistance to the $\mathrm{ABX}$ in use, reducing the selective pressure exerted on the microbial flora (Sandiumenge et al., 2003).

Preemptive treatment is the administration of $\mathrm{ABX}$ in some patients before clinical signs of infection appear. The application of this concept to critically ill patients is based on the identification of patients at risk of infections associated with high mortality, such as systemic fungal infections (Alvarez Lerma et al., 2010).

Having information on the quality of antibiotic prescription can help identify problems and implement strategies to improve this prescription; the European Surveillance of Antimicrobial Consumption (ESAC) has developed a group of indicators of antibiotic use in outpatients with the objective to measure the quality of the use of antibiotics and thus improve their use (Coenen et al., 2007).

\section{Conclusion}

Despite the fact that no prospective studies were identified in this SR, rationale and day-to-day clinical practice experience suggest that multidisciplinary participation of different specialists in ABX's Infection and Policy Commission (or the ABX Commission), or the Pharmacy and Therapeutics Committee, might improve the development and application of these strategies.
Funding: None.

\section{Conflicts of interest:}

The authors declare no potential conflicts of interest.

\section{Acknowledgements:}

Permission to reproduce UpToDate ${ }^{\circledR}$ material, and Cambridge University Press grant non-exclusive permission, free of charge, for table 2 from: Michael Klompas, Richard Branson, Eric C. Eichenwald, Linda R. Greene, Michael D. Howell, Grace Lee, Shelley S. Magill, Lisa L. Maragakis, Gregory P. Priebe, Kathleen Speck, Deborah S. Yokoe, Sean M. Berenholtz. (2014). Strategies to Prevent Ventilator-Associated Pneumonia in Acute Care Hospitals: 2014 Update. Infection Control \& Hospital Epidemiology, 35(8), 915-936 (c) 2014 by The Society for Healthcare Epidemiology of America, published by Cambridge University Press. 


\section{PRISMA 2009 Flow Diagram}

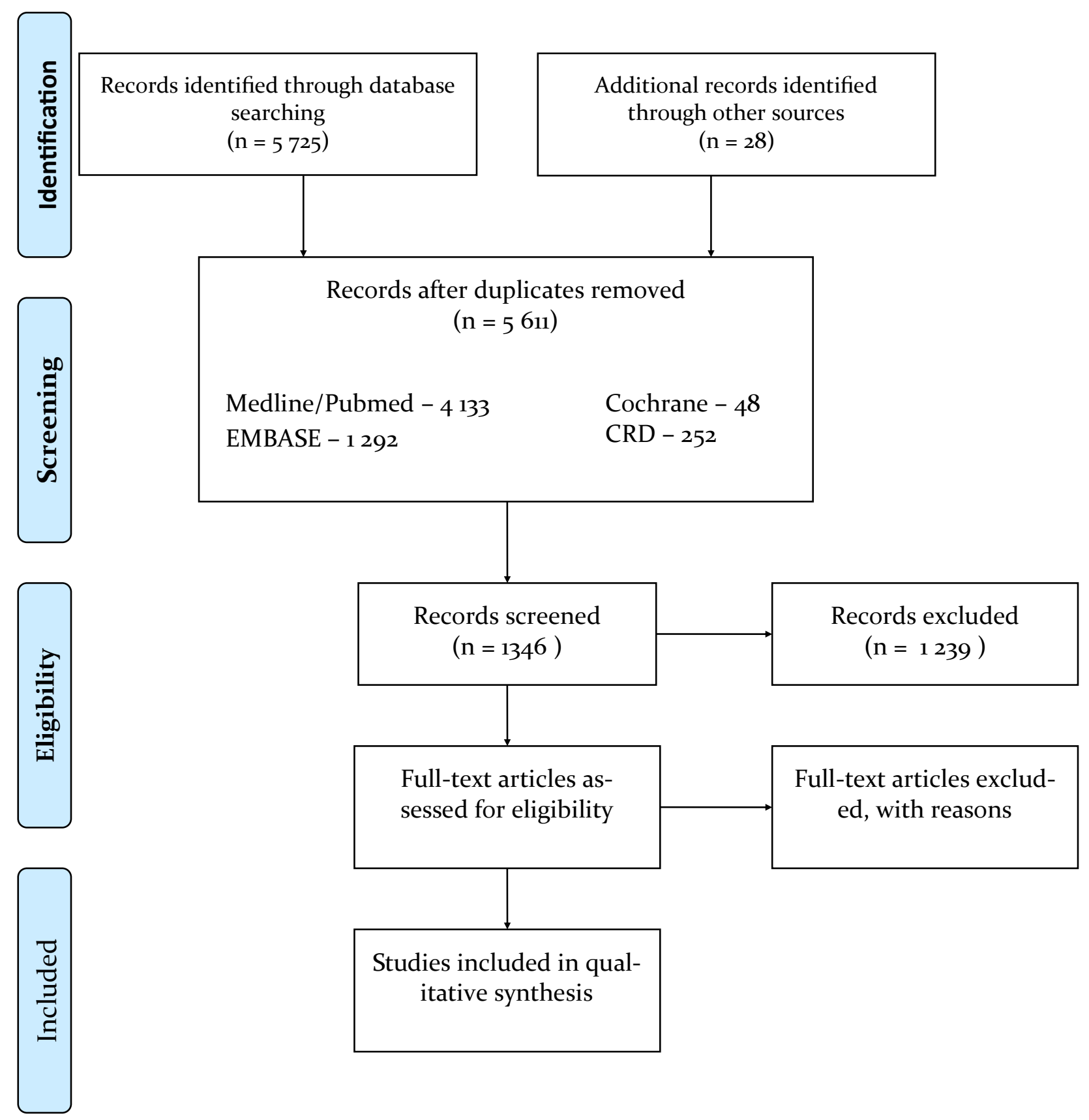

From: Moher D, Liberati A, Tetzlaff J, Altman DG, The PRISMA Group (2009). Preferred Reporting Items for Systematic Reviews and Meta-Analyses: The PRISMA Statement. PLoS Med 6(7): e1000097. doi:10.1371/ journal.pmedioooog7 


\section{Table 1. Summary of recommendations for preventing ventilator-associated pneumonia (VAP) in adult patients - Society for Healthcare Epidemiology of America/Infectious Diseases Society of America (SHEA/IDSA)}

$\begin{array}{ll}\text { Recommendation } & \text { Rationale } \\ \text { Basic practices } & \begin{array}{l}\text { Good evidence that the inter- } \\ \text { vention decreases the average } \\ \text { duration of mechanical ventila- } \\ \text { tion, length of stay, mortality, } \\ \text { and/or costs; benefits likely } \\ \text { outweigh risks }\end{array}\end{array}$

Special approaches

Generally not recommended

No recommendation

Good evidence that the interinsufficient data available on possible risks cient data to determine impact on duration of mechanical ventilation, length of stay, or mortality

Lowers VAP rates but ample tion of mechanical ventilation, length of stay, or mortality tion, length of stay, or mortality $\Delta$ vention improves outcomes but

May lower VAP rates but insuffidata suggest no impact on dura-

No impact on VAP rates, average duration of mechanical ventila-

No impact on VAP rates or other patient outcomes, unclear impact on costs

\section{Intervention}

Use noninvasive positive pressure ventilation in selected populations

Manage patients without sedation whenever possible

Interrupt sedation daily

Assess readiness to extubate daily

Perform spontaneous breathing trials with sedatives turned off

Facilitate early mobility

Utilize endotracheal tubes with subglottic secretion drainage ports for patients expected to require greater than 48 or 72 hours of mechanical ventilation

Change the ventilator circuit only if visibly soiled or malfunctioning

Elevate the head of the bed to 30 to $45^{\circ}$

Selective oral or digestive decontamination

Quality of

evidence

High

Moderate

High

High

High

Moderate

Moderate

High

Low*

High $\uparrow$

Regular oral care with chlorhexidine

Moderate

Prophylactic probiotics

Moderate

Ultrathin polyurethane endotracheal tube cuffs

Low

Automated control of endotracheal tube cuff

Low

pressure

Saline instillation before tracheal suctioning

Low

Mechanical tooth brushing

Low

Silver-coated endotracheal tubes

Moderate

Kinetic beds

Moderate

Prone positioning

Moderate

Stress ulcer prophylaxis

Moderate

Early tracheotomy

High

Monitoring residual gastric volumes

Moderate

Early parenteral nutrition

Moderate

Closed/in-line endotracheal suctioning

Moderate

* There are very little data on head-of-bed elevation, but it is classified as a basic practice because of its simplicity, ubiquity, low cost, and potential benefit.

I There are abundant data on the benefits of digestive decontamination but insufficient data on the long-term impact of this strategy on antimicrobial resistance rates.

$\Delta$ May be indicated for reasons other than VAP prevention. 
Agencia Española de Medicamentos y Productos Sanitarios (AEMPS). Web. Plan estratégico y de acción para reducir el riesgo de selección y diseminación de resistencias a los antibióticos 2014-2018. AEMPS. http://www.aemps.gob.es/publicaciones/publica/ docs/plan-estrategico-antibioticos.pdf. .Accessed Jan 2018

Alvarez Lerma F, Sierra Camerino R, Alvarez Rocha L, Rodriguez Colomo O. (2010). [Antibiotic policy in critical patients]. Med Intensiva. 34(9):60o-8.

Backman C, Zoutman DE, Marck PB. (2008). An integrative review of the current evidence on the relationship between hand hygiene interventions and the incidence of health care-associated infections. Am J Infect Control. 36(5):333-48.

Climo MW, Yokoe DS, Warren DK, Perl TM, Bolon M, Herwaldt LA, et al. (2013). Effect of daily chlorhexidine bathing on hospital-acquired infection. N Engl J Med. 368(6):53342.

Coenen S, Ferech M, Haaijer-Ruskamp FM, Butler CC, Vander Stichele RH, Verheij TJ, et al. (2007). European Surveillance of Antimicrobial Consumption (ESAC): quality indicators for outpatient antibiotic use in Europe. Qual Saf Health Care. 16(6):440-5.

De Waele JJ, Vogelaers D, Blot S, Colardyn F. (2003). Fungal infections in patients with severe acute pancreatitis and the use of prophylactic therapy. Clin Infect Dis. 37(2):20813.

Dellinger RP, Levy MM, Rhodes A, Annane D, Gerlach H, Opal SM, et al. (2013). Surviving sepsis campaign: international guidelines for management of severe sepsis and septic shock: 2012. Crit Care Med. 41(2):580-637.

European Centre for Disease Control/European Medicines Agency. ECDC/EMEA Joint Technical Report. EMEA/576176/2009: The bacterial challenge: time to react. Stockholm: $\quad$ EMEA 2009. http://ecdc.europa.eu/en/publications/ Publications/ogog TER The Bacterial Challenge Time to React.pdf. Accessed Jan 2018.

Fourrier F, Cau-Pottier E, Boutigny H, Roussel-Delvallez M, Jourdain M, Chopin C. (20oo). Effects of dental plaque antiseptic decontamination on bacterial colonization and nosocomial infections in critically ill patients. Intensive Care Med. 26(9):1239-47.

Garnacho Montero J, Alvarez Lerma F, Ramirez Galleymore P, Palomar Martinez M, Alvarez Rocha L, Barcenilla Gaite F, et al. (2015). Combatting resistance in intensive care: the multimodal approach of the Spanish ICU "Zero Resistance" program. Crit Care. 19:114.

Huang SS, Septimus E, Kleinman K, Moody J, Hickok J, Avery TR, et al. (2013). Targeted versus universal decolonization to prevent ICU infection. $N$ Engl J Med. 368(24):225565.

Kim JW, Chung J, Choi SH, Jang HJ, Hong SB, Lim CM, et al. (2012). Early use of imipenem/ cilastatin and vancomycin followed by de-escalation versus conventional antimicrobials without de-escalation for patients with hospital-acquired pneumonia in a medical ICU: a randomized clinical trial. Crit Care. 16(1):R28.

Lam OL, McGrath C, Bandara HM, Li LS, Samaranayake LP. (2012). Oral health promotion interventions on oral reservoirs of staphylococcus aureus: a systematic review. Oral Dis. 2012;18(3):244-54. 
Mok K, Christian MD, Nelson S, Burry L. (2014). Time to Administration of Antibiotics among Inpatients with Severe Sepsis or Septic Shock. Can J Hosp Pharm, 67(3):213-9.

Nijssen S, Fluit A, van d, V, Top J, Willems R, Bonten MJ. (2010). Effects of reducing betalactam antibiotic pressure on intestinal colonization of antibiotic-resistant gramnegative bacteria. Intensive Care Med. 36(3):512-9.

Pammi M, Eddama O, Weisman LE. (2011). Patient isolation measures for infants with candida colonization or infection for preventing or reducing transmission of candida in neonatal units. Cochrane Database Syst Rev. (11):CDoo6o68.

Pedreira ML, Kusahara DM, de Carvalho WB, Nunez SC, Peterlini MA. (2009). Oral care interventions and oropharyngeal colonization in children receiving mechanical ventilation. Am J Crit Care. 18(4):319-28.

Piarroux R, Grenouillet F, Balvay P, Tran V, Blasco G, Millon L, et al. (2004). Assessment of preemptive treatment to prevent severe candidiasis in critically ill surgical patients. Crit Care Med. 32(12):2443-9.

Rodriguez-Bano J, Pano-Pardo JR, Álvarez-Rocha L, Asensio A, Calbo E, Cercenado E, et al. (2012). [Programs for optimizing the use of antibiotics (PROA) in Spanish hospitals: GEIH-SEIMC, SEFH and SEMPSPH consensus document]. Farm Hosp. 36(1):33o.

Sandiumenge A, Rello J. (2003). [Cyclic rotation of antibiotics. Is all that glitters gold?]. Enferm Infecc Microbiol Clin. 21(2):93-100.

Scannapieco FA, Yu J, Raghavendran K, Vacanti A, Owens SI, Wood K, et al. (2009). A randomized trial of chlorhexidine gluconate on oral bacterial pathogens in mechanically ventilated patients. Crit Care. 13(4):R117.

Schuts EC, van den Bosch CM, Gyssens IC, Kullberg BJ, Hall MAL, Natsch S, et al. (2016). Adoption of a national antimicrobial guide (SWAB-ID) in the Netherlands. European Journal of Clinical Pharmacology. 72(2):249-52.

Silva BN, Andriolo RB, Atallah AN, Salomao R. (2013). De-escalation of antimicrobial treatment for adults with sepsis, severe sepsis or septic shock. Cochrane Database Syst Rev. 3:CDoo7934. doi: 10.1002/14651858.CDoo7934.pub3.

Slota M, Green M, Farley A, Janosky J, Carcillo J. (2001). The role of gown and glove isolation and strict handwashing in the reduction of nosocomial infection in children with solid organ transplantation. Crit Care Med. 29(2):405-12.

WHO Collaborating Centre for Drug Statistics Methodology. (2017). Guidelines for ATC classification and DDD assignment 2018. Oslo. 